\title{
A first-order version of Pfaffian closure
}

\author{
by
}

\author{
Sergio Fratarcangeli (New Rochelle, NY)
}

\begin{abstract}
The purpose of this paper is to extend a theorem of Speissegger [J. Reine Angew. Math. 508 (1999)], which states that the Pfaffian closure of an o-minimal expansion of the real field is o-minimal. Specifically, we display a collection of properties possessed by the real numbers that suffices for a version of the proof of this theorem to go through. The degree of flexibility revealed in this study permits the use of certain model-theoretic arguments for the first time, e.g. the compactness theorem. We illustrate this advantage by deriving a uniformity result on the number of connected components for sets defined with Rolle leaves, the building blocks of Pfaffian-closed structures.
\end{abstract}

1. Introduction. Let $\overline{\mathbb{R}}$ be the field of real numbers. Let $\widetilde{\mathbb{R}}$ be an o-minimal expansion of $\overline{\mathbb{R}}$. Unless stated otherwise, "definable" means "definable with parameters." Speissegger proves the following theorem in [Spe99]:

FACT 1.1. There is an o-minimal expansion $\mathfrak{P}(\widetilde{\mathbb{R}})$ of $\widetilde{\mathbb{R}}$ such that whenever $\omega$ is a $\mathfrak{P}(\widetilde{\mathbb{R}})$-definable 1 -form on a definable open set $U$ and $L$ is a Rolle leaf of $\omega=0$, then $L$ is definable in $\mathfrak{P}(\widetilde{\mathbb{R}})$.

The structure $\mathfrak{P}(\widetilde{\mathbb{R}})$ is called the Pfaffian closure of $\widetilde{\mathbb{R}}$ because in it the graphs of all Pfaffian functions are definable. It also follows from the theorem that o-minimality of $\widetilde{\mathbb{R}}$ is not destroyed by adjoining antiderivatives of definable functions.

On the other hand, this result is limited in that it only makes sense for expansions of $\overline{\mathbb{R}}$, as the proofs in [Spe99] make casual use of many special topological properties of the real line (e.g. the Baire category theorem, Heine-Borel theorem, and second countability). Indeed, the definition of "Rolle leaf" involves the notion of connectivity, a delicate property from the model-theoretic point of view.

2000 Mathematics Subject Classification: Primary 03C64; Secondary 58A17.

Key words and phrases: Pfaffian closure, o-minimal structures.

Research supported by NSERC. 
In this paper, we provide a notion of Pfaffian closure for o-minimal expansions of fields whose underlying universe is not necessarily the real line. Our proposed definition depends on a chosen expansion of the base structure that is definably complete. (This fact is unavoidable by Remark 2.8.) If the chosen expansion defines a set of integers in a sense that specializes that of Mourgues and Ressayre [MR93], then our Pfaffian closure is o-minimal. Though this result remains fairly restrictive, we use it to prove a new uniformity result on the number of connected components of definable sets in $\mathfrak{P}(\widetilde{\mathbb{R}})$.

Throughout, we let $\overline{\mathcal{R}}=(R,+, \cdot,<, 0,1)$ be a real closed ordered field, and let $\widetilde{\mathcal{R}}$ be an o-minimal expansion of $\overline{\mathcal{R}}$ to a language $\widetilde{\mathfrak{L}}$. We also view $\widetilde{\mathcal{R}}$ as a reduct of another structure $\mathcal{R}$, whose properties are described below. Here and throughout, we use the terms "expansion" and "reduct" in the sense of definability; that is, we assume that every $\overline{\mathcal{R}}$-definable set is $\widetilde{\mathcal{R}}$-definable and that every $\widetilde{\mathcal{R}}$-definable set is $\mathcal{R}$-definable. In case of ambiguity, the term "definable" refers to the structure $\mathcal{R}$.

Following Pillay and Steinhorn [PS86], we say $\mathcal{R}$ is definably complete if any definable nonempty subset of $R$ that is bounded above has a least upper bound. We henceforth assume that $\mathcal{R}$ is definably complete.

A subset $A$ of $R^{n}$ is definably connected if for each pair of disjoint open definable subsets $U$ and $V$ of $R^{n}$ such that $A=(A \cap U) \cup(A \cap V)$, we have either $A \cap U=\emptyset$ or $A \cap V=\emptyset$. (Note: we do not require that the set $A$ be definable.)

Definition 1.2. A definable subset $M$ of $R^{n}$ is an $\mathcal{R}$-manifold of dimension $m$ and class $C^{k}$ if for every $a$ in $M$ there are open subsets $U$ and $V$ of $R^{n}$ and a definable diffeomorphism $\varphi=\left(\varphi_{1}, \ldots, \varphi_{n}\right): U \rightarrow V$ of class $C^{k}$ (called a chart for $M$ at $a$ ) such that $a \in U, 0 \in V, \varphi(a)=0$, and

$$
U \cap M=\left\{x \in R^{n}: \varphi_{m+1}(x)=0, \ldots, \varphi_{n}(x)=0\right\} .
$$

In the case when $m=n$, this simply means that $M$ is a definable open subset of $R^{n}$.

Note that in case $R=\mathbb{R}$, an $\mathcal{R}$-manifold is just a definable embedded submanifold of $\mathbb{R}^{n}$ with definable charts. From now on, all $\mathcal{R}$-manifolds are assumed to be of class at least $C^{1}$.

Extending the notation above, we decompose a chart $\varphi$ into the functions $\bar{\varphi}=\left(\varphi_{1}, \ldots, \varphi_{m}\right)$ and $\widehat{\varphi}=\left(\varphi_{m+1}, \ldots, \varphi_{n}\right)$. Then the set $U \cap M$ is equal to the zero set of the definable function $\widehat{\varphi}$. In addition, for each $a$ in $M$ we define the tangent space $T_{a} M$ of $M$ at $a$ to be the vector subspace of $R^{n}$ given by

$$
T_{a} M:=\operatorname{ker} d_{a} \widehat{\varphi} .
$$

In the case $m=n$, we set $T_{a} M:=R^{m}$. This notion is well-defined, as is easily checked. 
We identify a 1-form

$$
\omega=a_{1}(x) d x_{1}+\cdots+a_{n}(x) d x_{n}
$$

on a subset of $\mathbb{R}^{n}$ with the vector field $F_{\omega}=\left(a_{1}(x), \ldots, a_{n}(x)\right)$ of its component functions. Furthermore, we say $\omega$ is nonsingular if $F_{\omega}$ is nowhere vanishing. From now on, all 1-forms are assumed to be nonsingular and of class $C^{1}$.

Definition 1.3. Let $\omega$ be a 1 -form on an open subset $U$ of $R^{n}$. We say that an $\mathcal{R}$-manifold of dimension $n-1$ and class $C^{1}$ is an integral $\mathcal{R}$-manifold of $\omega=0$ if $M$ is a subset of $U$ and $T_{a} M=\operatorname{ker}(\omega(a))$ for all $a$ in $M$. An $\mathcal{R}$-leaf of $\omega=0$ is a definably connected integral $\mathcal{R}$-manifold of $\omega=0$ that is relatively closed in $U$.

Definition 1.4. Let $\omega$ be a 1 -form on an open subset $U$ of $R^{n}$. An $\mathcal{R}$-leaf $L$ of $\omega=0$ is called Rolle if $L$ satisfies the following additional property:

[R-Rolle Property] For every definably connected $\mathcal{R}$-manifold $C$ of dimension 1 and class $C^{1}$ that is contained in $U$, either $|C \cap L| \leq 1$ or there is an $a$ in $C$ such that $T_{a} C \subseteq \operatorname{ker}(\omega(a))$.

For all natural numbers $i$, we define languages $\mathfrak{L}_{i}$ recursively as follows: Set $\mathfrak{L}_{0}=\widetilde{\mathfrak{L}}$. Then $\mathfrak{L}_{i+1}$ is the language obtained by adding to $\mathfrak{L}_{i}$, for each $\mathfrak{L}_{i}$-definable 1 -form $\omega$ and each Rolle $\mathcal{R}$-leaf $L$ of $\omega=0$, a predicate symbol for $L$. Define $\mathfrak{L}_{\text {Rolle }}$ to be the language

$$
\mathfrak{L}_{\text {Rolle }}:=\bigcup_{i \in \mathbb{N}} \mathfrak{L}_{i}
$$

and define the structure $\mathfrak{P}(\widetilde{\mathcal{R}}, \mathcal{R})$ to be the expansion of $\widetilde{\mathcal{R}}$ to the language $\mathfrak{L}_{\text {Rolle }}$. We call the structure $\mathfrak{P}(\widetilde{\mathcal{R}}, \mathcal{R})$ the relative Pfaffian closure of $\widetilde{\mathcal{R}}$ in $\mathcal{R}$.

Let $\mathbb{R}_{\text {proj }}:=(\overline{\mathbb{R}}, \mathbb{Z})$, and let $T_{\text {proj }}:=\operatorname{Th}\left(\mathbb{R}_{\text {proj }}\right)$. A set is definable in $\mathbb{R}_{\text {proj }}$ if and only if it is projective in the sense of descriptive set theory (see Kechris [Kec95] for example). In particular, every Borel subset of $\mathbb{R}^{n}$ is definable in $\mathbb{R}_{\text {proj }}$ for each $n$. By cell decomposition, it follows that every o-minimal expansion of $(\mathbb{R},<)$ is a reduct of $\mathbb{R}_{\text {proj }}$. Also every embedded submanifold of $\mathbb{R}^{n}$ and every 1-form on an open subset of $\mathbb{R}^{n}$ is definable in $\mathbb{R}_{\text {proj}}$. In this setting, the main theorem from [Spe99] can be reformulated as follows.

FACT 1.5. If $\widetilde{\mathbb{R}}$ is an o-minimal expansion of $\overline{\mathbb{R}}$, then $\mathfrak{P}\left(\widetilde{\mathbb{R}}, \mathbb{R}_{\text {proj }}\right)$ is o-minimal.

The main theorem of this paper is the following:

THEOREM 1.6. There is a recursive fragment $T$ of $T_{\text {proj }}$ such that if $\mathcal{R} \models T$, then $\mathfrak{P}(\widetilde{\mathcal{R}}, \mathcal{R})$ is o-minimal. 
The (admittedly verbose) axiomatization of $T$ is described in Section 4. On the other hand, we now exhibit an application of Theorem 1.6 to $\mathfrak{P}(\widetilde{\mathbb{R}})$, which can be derived without knowing the details of $T$.

We say that a manifold $L$ is a Rolle leaf of $F_{\omega}$ if $L$ is a Rolle $\mathbb{R}_{\text {proj-leaf }}$ of $\omega=0$.

Take $\widetilde{\mathfrak{L}}$ to be the language of $\widetilde{\mathbb{R}}$, and let $\mathcal{P}=\left(P_{1}, \ldots, P_{j}\right)$ be a finite tuple of predicate symbols that are not contained in $\widetilde{\mathfrak{L}}$. Also, let $\Phi:=\left(\phi_{0}, \ldots, \phi_{j}\right)$ be a finite tuple of $(\widetilde{\mathfrak{L}} \cup \mathcal{P})$-formulas, and let $X$ be a subset of $\mathbb{R}^{n}$ that is definable in $\mathfrak{P}(\widetilde{\mathbb{R}})$.

Definition 1.7. We say $X$ has format $\Phi$ if there is an interpretation of the predicates $P_{1}, \ldots, P_{j}$ such that the following hold:

(1) Each $\phi_{i}$ is in the language $\widetilde{\mathfrak{L}} \cup\left\{P_{1}, \ldots, P_{i}\right\}$.

(2) For $i=0, \ldots, j-1$, each $\phi_{i}$ defines a nonvanishing vector field on an open set $U_{i}$.

(3) For $i=0, \ldots, j-1$, each $P_{i+1}$ defines a Rolle leaf of the vector field defined by $\phi_{i}$.

(4) The set $X$ is defined by the formula $\phi_{j}$.

With this terminology, we derive the following consequence of Theorem 1.6:

TheOREM 1.8. For each $\Phi$ as above, there is a natural number $K$ such that whenever a set $X$ has format $\Phi$, the set $X$ has fewer than $K$ connected components.

Remark: The definition of the "format" of a set was inspired by Gabrielov's work in [Gab03]. There he defines the format of a "limit set" in order to derive an effective bound on the number of its components. In contrast, the bounds given by Theorem 1.8 are not effective. This suggests the following question:

Suppose the language $\widetilde{\mathfrak{L}}$ is recursive. Is there an algorithm that, given a format $\Phi$, provides a bound $K$ on the number of connected components of sets $X$ with format $\Phi$ ?

These results continue a thread of inquiry with roots in the work of Khovanski1 [Kho91]. Wilkie [Wi199] first proved that the real field expanded by Pfaffian functions is o-minimal. Other advances were made by Lion and Rolin [LR98] and Karpinsky and Macintyre [KM99]. In addition, the notion of a Rolle leaf was introduced by Moussu and Roche [MR91].

As our exposition is not self-contained, the reader is expected to be already familiar with [Spe99]. Instead of reproducing arguments at length, we focus only on areas that are most illustrative of the new difficulties that arise in our setting. 
Conventions. The letters $i, j, k, l, m, n, p, q$ range over natural numbers, and the letters $r, s, t$ range over $R$. Unless stated otherwise, the letters $x, y$, and $z$ denote tuples of variables $x=\left(x_{1}, \ldots, x_{n}\right), y=\left(y_{1}, \ldots, y_{m}\right)$ and $z=\left(z_{1}, \ldots, z_{l}\right)$ that range over $R^{n}, R^{m}$, and $R^{l}$ respectively.

Given vectors $v_{1}, \ldots, v_{k}$ in $R^{n}$, we write $\left\langle v_{1}, \ldots, v_{k}\right\rangle$ for the $R$-linear span of the set $\left\{v_{1}, \ldots, v_{k}\right\}$. We also let $\left\{e_{1}, \ldots, e_{n}\right\}$ be the standard basis for $R^{n}$.

Given an $r$ in $R$, we set $|r|:=\max \{r,-r\}$ and equip $R^{n}$ with the distance function

$$
d\left(x, x^{\prime}\right):=\max \left\{\left|x_{1}-x_{1}^{\prime}\right|, \ldots,\left|x_{n}-x_{n}^{\prime}\right|\right\} .
$$

For an element $a$ and definable nonempty subset $B$ of $R^{n}$, we define the distance from $a$ to the set $B$ by

$$
d(a, B):=\inf \{d(a, b): b \in B\} .
$$

We also use the notation $\|a\|$ to represent $d(a, 0)$. For positive $t$ in $R$, we let $B(a, t)$ denote $\left\{x \in R^{n}: d(x, a)<t\right\}$, the open ball of radius $t$ around $a$.

Let $A$ be a subset of $R^{n}$. We use $|A|$ to denote the cardinality of $A$. We write $\operatorname{cl}(A), \operatorname{int}(A)$, and $\operatorname{bd}(A):=\operatorname{cl}(A) \backslash \operatorname{int}(A)$ for the topological closure, interior, and boundary of $A$ respectively. We also define the sets

$$
\begin{aligned}
& T(A, t):=\left\{x \in R^{n}: d(x, a)<t \text { for some } a \in A\right\}, \\
& S(A, t):=\left\{x \in R^{n}: d(x, a) \leq t \text { for some } a \in A\right\} .
\end{aligned}
$$

For $B \subseteq R^{n+m}$, we let $B_{x}$ denote the fiber $\left\{y \in R^{m}:(x, y) \in B\right\}$ of $B$ over $x$.

An $\mathcal{R}$-manifold $M$ in $R^{n}$ is said to be in standard position if for every strictly increasing map $\iota:\{1, \ldots, k\} \rightarrow\{1, \ldots, n\}$ there is a number $d=$ $d(M, \iota)$ such that $\left.\Pi_{\iota}\right|_{M}$ has constant rank $d$.

2. Preliminaries. We begin by recalling some useful consequences of definable completeness. The structure $\mathcal{R}$ is said to have the intermediate value property if for all $a$ and $b$ in $R$, each continuous definable function $f:[a, b] \rightarrow R$ takes on every value in $R$ between $f(a)$ and $f(b)$. This notion is related to definable completeness as illustrated by the following proposition of C. Miller [Mil01].

Proposition 2.1. The following are equivalent:

(i) $\mathcal{R}$ is definably complete.

(ii) $\mathcal{R}$ has the intermediate value property.

(iii) $R$ is definably connected.

(iv) Intervals in $R$ are definably connected.

(v) If $f: A \rightarrow R^{n}$ is a definable continuous function and $A$ is a closed and bounded subset of $R^{m}$, then the set $f(A)$ is closed and bounded. 
(vi) If $f: A \rightarrow R$ is a definable continuous function and $A$ is a closed and bounded subset of $R^{n}$, then $f$ attains a max and min in $A$.

Proof. In fact [Mil01] only claims (i) $\Leftrightarrow($ ii $) \Leftrightarrow($ iii $) \Leftrightarrow($ iv $) \Leftrightarrow(v) \Rightarrow(v i)$. Here is the remaining implication, (vi) $\Rightarrow(\mathrm{i})$ : Let $A$ be a nonempty definable subset of $R$ that is bounded above, and assume for a contradiction that $\sup A$ does not exist. Define

$$
\widetilde{A}=\{t \in R: \exists s \in A, t<s\},
$$

so that $\widetilde{A}$ is a definable initial segment of $R$. Choose $a \in \widetilde{A}$ and $b \in R \backslash \widetilde{A}$ and define $f:[a, b] \rightarrow R$ by

$$
f(t)= \begin{cases}t & \text { if } t \in \widetilde{A}, \\ a & \text { if } t \notin \widetilde{A} .\end{cases}
$$

Since $\sup A$ does not exist, the function $f$ is continuous. By (vi), $\max f=$ $f(c)$ for some $c \in[a, b]$. But $c$ can be in neither $\widetilde{A}$ nor $R \backslash \widetilde{A}$.

Lemma 2.2. Let $A$ be a definable and definably connected subset of $R^{n}$. Let $B$ be a definable nonempty subset of $A$ that is both relatively open and closed in $A$. Then $B=A$.

Proof. Let $C=A \backslash B$, and suppose that $C \neq \emptyset$. Then the definable sets

$$
\begin{aligned}
& U:=\left\{a \in R^{n}: d(a, B)<d(a, C)\right\}, \\
& V:=\left\{a \in R^{n}: d(a, C)<d(a, B)\right\}
\end{aligned}
$$

contradict the fact that $A$ is definable connected.

REMARK 2.3. Though this lemma was predictable, it is unclear whether it holds in ordered structures that do not expand a densely ordered abelian group. It also shows that the (a priori different) definition of "definably connected" given in [vdD98] agrees in our setting with the definition above.

Definition 2.4. Let $A$ be a subset of $R^{n}$. Then a subset $C$ of $A$ is a definably connected component of $A$ (or just a component of $A$ for short) if $C$ is a maximal definably connected subset of $A$.

Definably connected components always exist by Zorn's lemma, and distinct components of a given set are disjoint. Though components need not be definable, we do get the following:

Proposition 2.5. If $A$ is a definable set with only finitely many components $C_{1}, \ldots, C_{l}$, then each $C_{i}$ is definable and both open and closed in $A$.

Proof. We show $C_{1}$ is definable and open in $A$. For each $j=\{2, \ldots, l\}$ there is a definable open set $U_{j}$ such that $U_{j} \cap\left(C_{1} \cup C_{j}\right)=C_{1}$. Then $C_{1}=\bigcap_{j=2}^{l} U_{j} \cap A$. 
In [vdD98], van den Dries proves some versions of theorems from calculus in the o-minimal setting. In fact, these proofs only use definable completeness. For example, we have the mean value theorem.

Theorem 2.6 (Mean Value Theorem). Suppose $a<b$ in $R$ and $f:[a, b]$ $\rightarrow R$ is a definable function. If the derivative $f^{\prime}(t)$ exists for all $t \in[a, b]$, then for some $c \in(a, b)$, we have $f(b)-f(a)=(b-a) f^{\prime}(c)$.

Using this, we get uniqueness for definable solutions of certain differential equations.

Proposition 2.7. Let $I$ and $J$ be open intervals in $R$. Suppose $f, g$ : $I \rightarrow J$ are definable functions of class $C^{1}$ on I such that the set $\{t \in I: f(t)$ $=g(t)\}$ is nonempty. Suppose also that $F: I \times J \rightarrow R$ is a definable function and that the partial derivative $D_{2} F$ exists and is continuous on $I \times J$. Suppose finally that $f^{\prime}(t)=F(t, f(t))$ and $g^{\prime}(t)=F(t, g(t))$ for all $t$ in $I$. Then $f=g$.

REMARK 2.8. As a special case, this implies that a DC expansion of a real closed field cannot define two distinct exponential functions. Kuhlmann and Shelah [KS05] show that for each regular uncountable cardinal $\kappa$ there is a real closed field that admits $2^{\kappa}$ pairwise nonisomorphic models of real exponentiation. Consequently, in any o-minimal expansion of such a field, at most one of these exponential functions is definable.

The proof of the proposition resembles that of Theorem 2.3 of Otero, Peterzil, and Pillay [OPP96], which takes place in the o-minimal setting. We include details to emphasize that definable completeness alone suffices. Here is a lemma.

Lemma 2.9. Suppose $I$ is an open interval in $R$. Suppose $u: I \rightarrow R$ is a definable function, $u^{\prime}(t)$ exists for all $t$ in $I$, and $u(t)=0$ for some $t$ in $I$. Suppose finally that for each $t_{0}$ in $I$ there is a neighborhood $V$ of $t_{0}$ and an $r$ in $R$ such that $\left|u^{\prime}(t)\right| \leq r|u(t)|$ for all $t$ in $V$. Then $u(t)=0$ for all $t$ in $I$.

Proof. Define $A$ to be the set $\{t \in I: u(t)=0\}$. By our assumptions, $A$ is nonempty, closed in $I$, and definable. By Lemma 2.2, it suffices to show that $A$ is open.

Fix $t_{0}$ in $A$. By replacing $u(t)$ by $u\left(t-t_{0}\right)$, we may assume $t_{0}=0$. Let $r$ and $V$ be as in our hypotheses, and choose $\varepsilon>0$ such that $[-\varepsilon, \varepsilon] \subseteq V$ and $r \varepsilon<1$. We show $[-\varepsilon, \varepsilon] \subseteq A$, which shows $A$ is open. Set $s:=\max \{|u(t)|$ : $t \in[-\varepsilon, \varepsilon]\}$. Suppose there is a $t \in(0, \varepsilon]$ such that $|u(t)|=s$. Then we can set

$$
t_{1}:=\inf \{t \in(0, \varepsilon]:|u(t)|=s\} .
$$

In this case, $\left|u\left(t_{1}\right)\right|=s$ by continuity. Suppose for a contradiction that $t_{1} \neq 0$. Then, by the Mean Value Theorem, there is a $t_{2} \in\left(0, t_{1}\right)$ such that 
$\left|u\left(t_{1}\right)\right|=\left|t_{1} u^{\prime}\left(t_{2}\right)\right|$. In this case we have

$$
s=\left|u\left(t_{1}\right)\right|=\left|t_{1} u^{\prime}\left(t_{2}\right)\right| \leq r\left|t_{1} u\left(t_{2}\right)\right|<\left|u\left(t_{2}\right)\right|,
$$

a contradiction. The case where there is a $t \in[-\varepsilon, 0)$ with $|u(t)|=s$ is done in the same way.

Proof of Proposition 2.7. Let $u: I \rightarrow R$ be the function defined by $u(t):=f(t)-g(t)$, and let $t_{0}$ be an arbitrary element of $I$. It suffices to find a $V$ and an $r$ as in the previous lemma. Choose an $\varepsilon>0$ such that the closed interval $I_{1}:=\left[t_{0}-\varepsilon, t_{0}+\varepsilon\right]$ is contained in $I$. Set $a:=\min \left(f\left(I_{1}\right) \cup g\left(I_{1}\right)\right)$, $b:=\max \left(f\left(I_{1}\right) \cup g\left(I_{1}\right)\right)$, and $J_{1}:=[a, b]$. Consequently, if we take $V=$ $\left(t_{0}-\varepsilon, t_{0}+\varepsilon\right)$ and $r=\max _{I_{1} \times J_{1}}\left|D_{2} F\right|$, then we are done: For $t \in V$,

$$
\left|u^{\prime}(t)\right|=\left|(f-g)^{\prime}(t)\right|=|F(t, f(t))-F(t, g(t))| \leq r|f(t)-g(t)|=r|u(t)|
$$

by the mean value theorem.

Definable completeness allows us to prove versions of many other classical results from elementary differential geometry in the definable $C^{1}$ setting. This is the case of the inverse function theorem, the rank theorem, the Lagrange multipliers theorem, etc. For example, we can state the rank theorem as follows:

FACT 2.10 (Rank Theorem). Suppose $U$ and $V$ are definable open subsets of $R^{n}$ and $R^{m}$ respectively and that $f: U \rightarrow V$ is a definable function of class $C^{1}$ with constant rank $d$. Then for any a in $U$ there exist definable open neighborhoods $U^{\prime}$ of a and $V^{\prime}$ of $f(a)$, open subsets $\widetilde{U}$ of $R^{n}$ and $\widetilde{V}$ of $R^{m}$, and definable diffeomorphisms $\varphi: U^{\prime} \rightarrow \widetilde{U}$ and $\psi: V^{\prime} \rightarrow \widetilde{V}$ of class $C^{1}$ such that

$$
\psi \circ f \circ \varphi^{-1}\left(x_{1}, \ldots, x_{d}, x_{d+1}, \ldots, x_{n}\right)=\left(x_{1}, \ldots, x_{d}, 0, \ldots, 0\right) .
$$

We close this section with two technical lemmas adapted from [Spe99]. Let $U$ be an open subset of $R^{n}$, and fix a finite tuple $\Omega:=\left(\omega_{1}, \ldots, \omega_{q}\right)$ of $\widetilde{\mathcal{R}}$-definable 1 -forms $\omega_{i}$ on $U$. For $i=1, \ldots, q$ we also write $F_{i}$ for the vector field $F_{\omega_{i}}$ associated with $\omega_{i}$.

Definition 2.11. Let $N$ be an $\mathcal{R}$-manifold contained in $U$. The tuple $\Omega$ is transverse to $N$ if

$$
\operatorname{dim}\left(T_{a} N \cap \bigcap_{i=1}^{q} \operatorname{ker}\left(\omega_{i}(a)\right)\right)=\operatorname{dim}(N)-q
$$

for all $a \in N$. If $N=R^{n}$, we simply say $\Omega$ is transverse. For a subset $J$ of $\{1, \ldots, q\}$, we write $\Omega_{J}:=\left(\omega_{j}\right)_{j \in J}$. The tuple $\Omega_{J}$ is called a basis of $\Omega$ along $N$ if $\Omega_{J}$ is transverse to $N$ and

for all $a \in N$.

$$
T_{a} N \cap \bigcap_{i=1}^{q} \operatorname{ker}\left(\omega_{i}(a)\right)=T_{a} N \cap \bigcap_{i \in J} \operatorname{ker}\left(\omega_{i}(a)\right)
$$


Lemma 2.12. Let $A$ be an $\widetilde{\mathcal{R}}$-definable subset of $R^{n}$. Then for any natural number $k$, there is a decomposition $\mathcal{P}$ of $R^{n}$ into $C^{k}$-cells such that $\mathcal{P}$ is compatible with both $A$ and $U$ and has the following property: Whenever a cell $C$ in $\mathcal{P}$ is a subset of $A$ and whenever $J$ is a subset of $\{1, \ldots, q\}$, there exists a subset $J^{\prime}$ of $J$ such that $\Omega_{J^{\prime}}$ is a basis of $\Omega_{J}$ along $C$.

Proof. See Lemmas 2.1 and 2.8 from [Spe99].

We now discuss the pullback of a 1-form.

DeFinition 2.13. Let $U$ and $V$ be open subsets of $R^{n}$, and let $\sigma: V \rightarrow U$ be a definable diffeomorphism. Suppose that $\omega$ is a definable 1-form on $U$. Then the pullback $\sigma^{*} \omega$ is the 1-form on $V$ given by

$$
\sigma^{*} \omega(a) v:=\omega(\sigma(a)) d_{a} \sigma v
$$

for all $a \in V$ and $v \in T_{a} V$.

Lemma 2.14. Let $U$ and $V$ be definable open subsets of $R^{n}$, and let $\sigma: V \rightarrow U$ be a definable diffeomorphism. Whenever $L_{i}$ is a Rolle $\mathcal{R}$-leaf of $\omega_{i}=0$, then $\sigma^{-1}\left(L_{i}\right)$ is a Rolle $\mathcal{R}$-leaf of $\sigma^{*} \omega_{i}=0$. Moreover, if $N$ is an $\mathcal{R}$-manifold contained in $U$ such that $\Omega$ is transverse to $N$, then the tuple of pullbacks $\sigma^{*} \Omega:=\left(\sigma^{*} \omega_{1}, \ldots, \sigma^{*} \omega_{q}\right)$ is transverse to $\sigma^{-1}(N)$.

Proof. Immediate from the definitions.

3. Khovanskiı̌ theory with definable completeness. The first step in proving o-minimality of our Pfaffian closure is to prove a suitable Khovanskil theory. It turns out that the assumption of definable completeness suffices for this task. Let $\Omega=\left(\omega_{1}, \ldots, \omega_{l}\right)$ be a tuple of $\widetilde{\mathcal{R}}$-definable (nonsingular) 1-forms defined on a common open subset $U$ of $R^{n}$. Here is the statement:

THEOREM 3.1. Let $A$ be an $\widetilde{\mathcal{R}}$-definable subset of $U$. Then there is a natural number $K$ such that whenever $L_{i}$ is a Rolle $\mathcal{R}$-leaf of $\omega_{i}=0$ for each $i=1, \ldots, l$, the set $A \cap L_{1} \cap \cdots \cap L_{l}$ is a union of fewer than $K$ definably connected $\mathcal{R}$-manifolds.

Let us first loosely describe why a naive application of the existing proof fails: Arguing inductively, we would like to find a set $B$ of dimension lower than $A$ such that $B^{\prime}:=B \cap L_{1} \cap \cdots \cap L_{l}$ has at least as many components as $A^{\prime}:=A \cap L_{1} \cap \cdots \cap L_{l}$ for all $L_{i}$. To define $B$, we wish to find a definable function $\mu: A \rightarrow R$ that assumes a minimum on each component of $A^{\prime}$. Unfortunately, this argument requires that each component of $A^{\prime}$ be definable, which will not be clear until the end of the proof. To get around this problem, we integrate Proposition 2.5 into the induction, most evidently through a new case in Proposition 3.6 below. Now some lemmas. 
Lemma 3.2. Suppose $\omega$ is an $\widetilde{\mathcal{R}}$-definable 1 -form on $U$. Let $L$ be an integral $\mathcal{R}$-manifold of $\omega=0$ that is closed in $U$. Suppose a subset $C$ of $U$ is a definably connected $\mathcal{R}$-manifold of dimension at most $n-1$ and that $T_{x} C \subseteq \operatorname{ker}(\omega(x))$ for all $x$ in $C$. Then either $C \cap L=\emptyset$ or $C \subseteq L$.

This proof closely follows the proof of Lemma 1.4 in [Spe99]. However, because Lemma 2.2 is used, the definability of $C$ is essential.

Lemma 3.3. Let $N$ be an $\mathcal{R}$-manifold contained in $U$ such that $\Omega_{J}$ is a basis of $\Omega$ along $N$. For each $i=1, \ldots, q$, let $L_{i}$ be a definably connected integral $\mathcal{R}$-manifold of $\omega_{i}=0$, and write

$$
W:=N \cap \bigcap_{i \in 1}^{q} L_{i} \quad \text { and } \quad W_{J}:=N \cap \bigcap_{i \in J} L_{i} .
$$

Then $W_{J}$ is either empty or an $\mathcal{R}$-manifold of dimension $\operatorname{dim}(N)-|J|$. Moreover, if $W_{J}$ has only finitely many components, then each component of $W$ is a component of $W_{J}$ and an $\mathcal{R}$-manifold of dimension $\operatorname{dim}(N)-|J|$.

Proof. This follows the argument of Lemma 1.6 in [Spe99]. However, we have added a hypothesis in the "Moreover" statement; the new assumption that $W_{J}$ has finitely many components is necessary to conclude that each component is definable, and thus that Lemma 3.2 above can be applied.

The next lemma is used to lower the dimension in the inductive proof of Theorem 3.1.

Definition 3.4. Let $M=R^{m} \times\{0\}^{n-m}$. A positive $\widetilde{\mathcal{R}}$-form for $M$ is an $\widetilde{\mathcal{R}}$-definable continuous function $\mu: R^{n} \rightarrow[0, \infty)$ such that for each positive $r$, the set $M \cap \mu^{-1}([0, r])$ is bounded in $R^{n}$.

LemmA 3.5. Let $M=R^{m} \times\{0\}^{n-m}$. Suppose that $M$ is contained in $U$ and that $\Omega$ is transverse to $M$. Suppose also that $q<m$. Then there is a positive $\widetilde{\mathcal{R}}$-form $\mu$ for $M$ of class $C^{1}$ such that the definable set

$$
B:=\left\{a \in M: \nabla \mu(a) \in\left\langle F_{1}(a), \ldots, F_{q}(a), e_{m+1}, \ldots, e_{n}\right\rangle\right\}
$$

has dimension strictly less than $m$.

Proof. Similar to Lemma 2.5 in [Spe99].

Proposition 3.6. Let $N$ be an $\widetilde{\mathcal{R}}$-definable $C^{1}$-cell of dimension $m$ contained in $U$. Suppose that $q<m$ and that $\Omega$ is transverse to $N$. Then there is an $\widetilde{\mathcal{R}}$-definable closed subset $B$ of $N$ with $\operatorname{dim}(B)<m$ such that whenever $L_{i}$ is a Rolle $\mathcal{R}$-leaf of $\omega_{i}=0$ for each $i$, we have one of the following cases: Either

CASE 1. $N \cap L_{1} \cap \cdots \cap L_{q}$ has finitely many components, each meeting $B$, or 
CASE 2. $N \cap L_{1} \cap \cdots \cap L_{q}$ has infinitely many components, and infinitely many of them meet $B$.

Proof. First note that by Lemma 3.3, the set $N \cap L_{1} \cap \cdots \cap L_{q}$ is always either empty or an $\mathcal{R}$-manifold of dimension $m-q$. Since $N$ is a $C^{1}$-cell, we may assume without loss of generality that $N=R^{m} \times\{0\}^{n-m}$. Let $B$ and $\mu$ be the set and positive $\widetilde{\mathcal{R}}$-form for $N$ given by Lemma 3.5 . We claim that this $B$ works:

Suppose first that $N \cap L_{1} \cap \cdots \cap L_{q}$ has finitely many components. In this case we may apply Proposition 2.5 to conclude that each component $C$ is definable and closed in $N$. If we choose $r>0$, then $C \cap \mu^{-1}[0, r]$ is closed and bounded in $R^{n}$. Thus $\left.\mu\right|_{C}$ assumes a minimum value, say at $a$. This implies that

$$
\nabla \mu_{u}(a) \in T_{a}\left(N \cap L_{1} \cap \cdots \cap L_{q}\right)^{\perp}=\left\langle F_{1}(a), \ldots, F_{q}(a), e_{m+1}, \ldots, e_{n}\right\rangle,
$$

finishing this case.

Now suppose $N \cap L_{1} \cap \cdots \cap L_{q}$ has infinitely many definably connected components. We recursively produce infinitely many distinct components $C_{1}, C_{2}, \ldots$, each meeting $B$, and definable sets $V_{0} \supsetneq V_{1} \supsetneq \cdots$ with the following properties:

(1) Each $V_{i}$ is an open and closed subset of $N \cap L_{1} \cap \cdots \cap L_{q}$.

(2) Each $V_{i}$ contains infinitely many definably connected components of $N \cap L_{1} \cap \cdots \cap L_{q}$.

(3) $C_{i} \cap V_{j}=\emptyset$ whenever $j>i$.

To start, put $V_{0}:=N \cap L_{1} \cap \cdots \cap L_{q}$. Given $V_{i}$, we show how to produce $V_{i+1}$. Since $V_{i}$ is not definably connected, there are definable open disjoint $U_{1}$ and $U_{2}$ such that $V_{i} \subseteq U_{1} \cup U_{2}$ and both $V_{i} \cap U_{1}$ and $V_{i} \cap U_{2}$ are nonempty. Now one of $U_{1}$ or $U_{2}$, say $U_{2}$, contains infinitely many components of $N \cap L_{1} \cap$ $\cdots \cap L_{q}$. Set $V_{i+1}:=V_{i} \cap U_{2}$. Now $V_{i} \cap U_{1}$ is a closed subset of $N \cap L_{1} \cap \cdots \cap L_{q}$. Thus $\left.\mu\right|_{V_{i} \cap U_{1}}$ assumes a minimum value. As in the previous case, $B$ meets $V_{i} \cap U_{1}$. Thus $B$ meets some component $C$ of $V_{i} \cap U_{1}$. Take $C_{i+1}:=C$ and we are done.

We are now ready for the proof of Theorem 3.1:

Proof of Theorem 3.1. As in [Spe99], we use induction on $d:=\operatorname{dim}(A)$ and $q$. Assume $d>0$ and $q>0$, and that the result holds for lower values of $d$ or $q$. By Lemmas 2.12 and 3.3, it suffices to consider the case that $A$ is an $\widetilde{\mathcal{R}}$-definable $C^{1}$-cell contained in $U$ and $\Omega$ is transverse to $A$. Note that then $d \geq q$. For $i=1, \ldots, q$, we let $L_{i}$ be a definable Rolle $\mathcal{R}$-leaf of $\omega_{i}=0$, and we put $L:=L_{1} \cap \cdots \cap L_{q}$. There are two cases, each containing an item worthy of note. 
CASE $d>q$. Let $B$ be a closed definable subset of $A$ with the property described in Proposition 3.6. By the inductive hypothesis there is a natural number $K$, independent of the particular Rolle $\mathcal{R}$-leaves chosen, such that $B \cap L$ has fewer than $K$ components. Consequently, we must be in Case 1 of Proposition 3.6; so $A \cap L$ has only finitely many components, each meeting $B$. It follows that $A \cap L$ has fewer than $K$ components, and, by Lemma 3.3, each is an $\mathcal{R}$-manifold.

CASE $d=q$. Put $L^{\prime}:=L_{1} \cap \cdots \cap L_{q-1}$, and notice that $\operatorname{dim}\left(A \cap L^{\prime}\right)=1$. By the inductive hypothesis, there is a natural number $K$ (depending on $\Omega$ and $A$ but not on the leaves $L_{i}$ ) such that the $\mathcal{R}$-manifold $A \cap L^{\prime}$ has fewer than $K$ components. Let $C$ be one; observe that it is an $\mathcal{R}$-manifold (definable by Proposition 2.5). Now if $\left|C \cap L_{q}\right|>1$, then there is an $a$ in $C$ such that $T_{a} C \subseteq \operatorname{ker}\left(\omega_{q}(a)\right)$ by the $\mathcal{R}$-Rolle property. This contradicts $\omega_{q}$ 's transversality to $A \cap L^{\prime}$, so $\left|C \cap L_{q}\right| \leq 1$ for each component $C$ of $A \cap L^{\prime}$. Consequently, $\left|A \cap L^{\prime} \cap L_{q}\right|<K$.

REMARK 3.7. Having just used the $\mathcal{R}$-Rolle property, we explain why we defined it in terms of tangent spaces, as opposed to a more direct generalization involving definable $C^{1}$-curves $\gamma:[0,1] \rightarrow U$. We were given a 1-dimensional definably connected $\mathcal{R}$-manifold $C$. To obtain a curve $\gamma$, we need to know that $C$ is "definably path connected," which is unclear in general. On the other hand, the $\mathbb{R}_{\text {proj-Rolle property does agree with the }}$ definition from [Spe99] since every $C^{1}$-curve $\gamma:[0,1] \rightarrow \mathbb{R}^{n}$ is definable in $\mathbb{R}_{\text {proj }}$.

4. A recursive fragment of $T_{\text {proj }}$. As mentioned in the introduction, we do not need the full strength of $T_{\text {proj }}$ to prove that $\mathfrak{P}(\widetilde{\mathcal{R}}, \mathcal{R})$ is o-minimal. In this section, we describe a recursively axiomatizable fragment $T$ of $T_{\text {proj }}$ which suffices for Theorem 1.6. Our system is hardly optimal, but it is not our purpose to eliminate redundant axioms here - only to present a starting point for doing so.

Our language $\mathfrak{L}_{\text {proj }}=(+, \cdot,<, 0,1, \mathcal{Z})$ is the language of rings expanded by a single unary predicate $\mathcal{Z}$. A model $\mathcal{R}$ of $T$ is a definably complete expansion of a real closed field $\overline{\mathcal{R}}$ by an integer part $\mathcal{Z}$ - that is, $\mathcal{Z}$ is a closed discrete subring of $R$, and for all $r \in R$ there is a unique $z \in \mathcal{Z}$ such that $z \leq r<z+1$. (See [MR93].) Observe that this can be expressed in a recursive axiom scheme. (Proposition 2.1 suggests several ways of axiomatizing definable completeness.) We also require that $\mathcal{R}$ satisfy items (A) through $(\mathrm{H})$ below. We begin with some observations and conventions.

Let $\mathcal{N}:=\{z \in \mathcal{Z}: z \geq 0\}$. Already from definable completeness, we can deduce that $\mathcal{N}$ satisfies induction: Suppose $X$ is a definable subset of $\mathcal{N}$, that whenever $s \in X$ it is also true that $s+1 \in X$, and that $0 \in X$; then 
$X=\mathcal{N}$. Otherwise, the definable clopen set $\{r \in R: \exists z \in \mathcal{N} \backslash X(z<r)\}$ is nonempty.

Next observe that the fraction field $\mathcal{Q}$ of $\mathcal{Z}$ is dense in $R$; elements of $\mathcal{Q}$ will be called $\mathcal{R}$-rationals, and a cartesian product of intervals with $\mathcal{R}$-rational endpoints is called an $\mathcal{R}$-rational box.

A definable subset $A$ of $R^{n}$ is called $\mathcal{R}$-countable if $A$ is empty or there is a definable surjection $f: \mathcal{N} \rightarrow A$. The set $A$ is called $\mathcal{R}$-finite if $A$ is empty or there is a definable surjection $f:\{0,1, \ldots, \beta\} \rightarrow A$ for some $\beta$ in $\mathcal{N}$. A set that is not $\mathcal{R}$-finite is $\mathcal{R}$-infinite. As usual the set $\mathcal{Q}$ is $\mathcal{R}$-countable.

Let $J$ be a definable subset of $\mathcal{N}$. Then a definable subset $X$ of $J \times R^{n}$ is called a definable sequence in $R^{n}$. We shall denote such a collection by $\left(X_{\alpha}\right)_{\alpha \in J}$ or just $\left(X_{\alpha}\right)_{\alpha}$ with the understanding that there is a definable set $J \subseteq \mathcal{N}$ over which $\alpha$ ranges. Occasionally, we shall also denote a definable sequence by $(X(\alpha))_{\alpha}$. If $\widetilde{J}$ is a subset of $J$, then $X \cap(\widetilde{J} \times R)$ is called a definable subsequence of $\left(X_{\alpha}\right)_{\alpha \in J}$. A definable sequence $\left(X_{\alpha}\right)_{\alpha}$ is called terminating if there is a $\beta$ in $\mathcal{N}$ such that $X_{\alpha}=\emptyset$ whenever $\alpha \geq \beta$. We also use $\left(X_{\alpha}\right)_{\alpha<\beta}$ to denote such a sequence. If, on the other hand, the set $\left\{\alpha \in J: X_{\alpha} \neq \emptyset\right\}$ is unbounded, then we say the sequence $\left(X_{\alpha}\right)_{\alpha}$ is nonterminating. A definable sequence of sets is said to be increasing (resp. decreasing) if whenever $\alpha$ and $\beta$ are in $J$ and $\alpha<\beta$, then $X_{\alpha} \subseteq X_{\beta}$ (resp. $X_{\beta} \subseteq X_{\alpha}$ ).

For two definable closed and bounded nonempty subsets $V$ and $W$ of $R^{n}$, the Hausdorff distance $d(V, W)$ is defined by

$$
d(V, W):=\max \{\inf \{d(x, V): x \in W\}, \inf \{d(x, W): x \in V\}\} .
$$

If it exists, the $\operatorname{limit} \lim X_{\alpha}$ of a definable sequence $\left(X_{\alpha}\right)_{\alpha}$ in the induced topology is called the Hausdorff limit of $\left(X_{\alpha}\right)_{\alpha}$.

Lastly, we point out an important fact about $\mathbb{R}_{\text {proj: }}$ for each natural number $n$, there is a (parameter-free) definable family $X^{n} \subset \mathbb{R}^{n+1}$ such that the collection $\left\{X_{r}^{n}: r \in \mathbb{R}\right\}$ is equal to the collection of all subsets of $\mathbb{Z}^{n}$. (One way to prove this is to code subsets of $\mathbb{Z}^{n}$ into decimal expansions of reals.) The family $X^{n}$ is defined by some $\mathfrak{L}_{\text {proj-formula }} \phi_{n}(x, t)$. We use this fact to express axiom scheme (A).

(A) For each natural number $n$, the formula $\phi_{n}(x, t)$ defines a family $X^{n} \subset R^{n+1}$ such that the collection $\left\{X_{r}^{n}: r \in R\right\}$ is equal to the collection of all definable subsets of $\mathcal{Z}^{n}$. More precisely, for each $n$ and each $\mathfrak{L}_{\text {proj-formula }} \psi(x, y)$, we have an axiom that says:

$$
\forall y \exists t \forall x\left[\phi_{n}(x, t) \leftrightarrow\left(\bigwedge_{i=1}^{n} x_{i} \in \mathcal{Z} \wedge \psi(x, y)\right)\right] .
$$

We will not be as explicit with the other axioms. Before proceeding, we point out some consequences of our axioms so far. 
Proposition 4.1.

(1) There is a parameter-free definable subset $Y^{n}$ of $R^{n+1}$ such that $\left\{Y_{r}^{n}: r \in R\right\}$ is equal to the collection of all definable open subsets of $R^{n}$. Consequently, the same is true with "open" replaced by "closed."

(2) Let $m \geq 1$. There is a parameter-free definable subset $Z^{m, n}$ of $R^{m+n+1}$ such that $\left\{Z_{r}^{m, n}: r \in R\right\}$ is equal to the collection of all graphs of definable continuous functions from $R^{m}$ to $R^{n}$. Moreover, the same is true for all definable functions from $\mathcal{N}^{m}$ to $R^{n}$.

Proof. For (1), we use the fact that the $\mathcal{R}$-rational boxes form a basis for the topology on $R^{n}$ : Let $X^{4 n}$ be as in (A) above. Let $Y^{n}$ be the set given by

$Y^{n}:=\left\{\left(s_{0}, \ldots, s_{n-1}, r\right) \in R^{n+1}:\right.$ There is some $\left(m_{0}, \ldots, m_{4 n-1}\right)$ in $X_{r}^{4 n}$ such that $m_{4 i}<m_{4 i+1} s_{i}$ and $m_{4 i+2} s_{i}<m_{4 i+3}$ for $\left.i=0, \ldots, n-1\right\}$.

This shows (1). The fact that the graphs of continuous functions are closed implies (2).

REMARK 4.2. The previous proposition has many useful consequences. For example, it follows that one can express that a definable set is definably connected with a single first order sentence. As another example, we note that given a definable sequence $\left(X_{\alpha}\right)_{\alpha}$, the collection of Hausdorff limits of subsequences comprises a definable family.

Using the above observations, one can express the remaining properties $(\mathrm{B})-(\mathrm{H})$ in a first-order way. We leave the details to the reader.

(B) Let $\kappa$ and $\beta$ be in $\mathcal{N}$. If $f:\{0, \ldots, \kappa \beta\} \rightarrow\{0, \ldots, \beta-1\}$ is a definable (total) function, then there is a $\gamma \in\{0, \ldots, \beta-1\}$ and a definable injection $g:\{0, \ldots, \kappa\} \rightarrow f^{-1}(\gamma)$.

(C) If $\left(X_{\alpha}\right)_{\alpha \in J}$ is a definable sequence of $\mathcal{R}$-countable sets, then the union $\bigcup_{\alpha \in J} X_{\alpha}$ is $\mathcal{R}$-countable.

(D) For any unbounded subset $J$ of $\mathcal{N}$, any definable function $\varrho: J \rightarrow$ $(0, \infty)$ that satisfies $\liminf _{\alpha \rightarrow \infty} \varrho(\alpha)=0$, and any $r>1$, there is a strictly increasing definable function $\delta: \mathcal{N} \rightarrow J$ such that for all $\alpha$ in $\mathcal{N}$, we have $r \varrho(\delta(\alpha+1))<\varrho(\delta(\alpha))$. Note: it follows that the composite function $\varrho \circ \delta: \mathcal{N} \rightarrow(0, \infty)$ is strictly decreasing, and $\lim _{\alpha \rightarrow \infty} \varrho \circ \delta(\alpha)=0$.

(E) If $W$ is a definable subset of $R^{n}$ that is closed and bounded, and if $\left(U_{\alpha}\right)_{\alpha}$ is an increasing sequence of open subsets such that $W \subset \bigcup_{\alpha} U_{\alpha}$, then there exists a $\gamma \in \mathcal{N}$ such that $W \subset U_{\gamma}$.

(F) If $\left(Y_{\alpha}\right)_{\alpha}$ is a definable sequence of nowhere dense sets, then the union $\bigcup_{\alpha} Y_{\alpha}$ has empty interior. 
(G) Suppose $B$ is a bounded box in $R^{n}$. For each $p=1, \ldots, K$, let $Y^{p}$ be a definable subset of $(\mathcal{N})^{2} \times B$ such that for all $\alpha$ and $\beta$ in $\mathcal{N}$, the set $Y^{p}(\alpha, \beta):=Y_{(\alpha, \beta)}^{p}$ is closed. Then there are unbounded definable subsets $I$ and $J$ of $\mathcal{N}$ such that the following hold:

(i) For each $p$ and $\alpha \in I$, the definable sequence $\left(Y^{p}(\alpha, \beta)\right)_{\beta \in J}$ converges to a Hausdorff limit $Y^{p}(\alpha)$ if the sequence $\left(Y^{p}(\alpha, \beta)\right)_{\beta \in J}$ is nonterminating. In case this sequence is terminating, we set $Y^{p}(\alpha):=\emptyset$.

(ii) For each $p$, the definable sequence $\left(Y^{p}(\alpha)\right)_{\alpha \in I}$ converges to a Hausdorff limit $Y^{p}$ whenever $\left(Y^{p}(\alpha)\right)_{\alpha \in I}$ is nonterminating.

(H) Let $m \geq 1$, and let $Y(\alpha)_{\alpha}$ be a definable sequence of nonempty closed subsets of $[-1,1]^{m}$ converging to a Hausdorff limit $Y$. Assume that $a \in R$ is such that $Y_{a} \neq \emptyset$ and $Y_{a}$ is not a Hausdorff limit of $\left(Y(\alpha)_{a}\right)_{\alpha}$. Then there is a terminating definable sequence $\left(U_{\gamma}\right)_{\gamma<\beta}$ of $\mathcal{R}$-rational boxes in $[-1,1]^{m-1}$ such that

(*) $Y_{a} \cap U_{\gamma} \neq \emptyset$ for each $\gamma<\beta$, and for all sufficiently large $\alpha$, there is a $\gamma$ such that $Y(\alpha)_{a} \cap U_{\gamma}=\emptyset$.

Most of the axioms above, when translated into a statement about $\mathbb{R}_{\text {proj }}$ are classical: $(\mathrm{B})$ is a pigeonhole principle, $(\mathrm{C})$ says that a countable union of countable sets is countable, $(\mathrm{E})$ is the Heine-Borel theorem, and $(\mathrm{F})$ is the Baire category theorem. Axiom (D) simply says that, given a limit point of a sequence, we can choose a monotone convergent subsequence that converges at a specified rate.

To see that $(G)$ holds in $\mathbb{R}_{\text {proj }}$, first note that a bounded sequence of compact sets in $\mathbb{R}^{n}$ has a convergent subsequence in the Hausdorff topology. (See [Mun75, item 7, p. 279].) The fact that, given a countable family of such sequences, we can find a common set of indices along which each member converges, is proved by applying a diagonal argument of the kind used in the Ascoli-Arzelà theorem. (See [Roy88, Lemma 37, p. 167].)

Finally, that $(\mathrm{H})$ holds for $\mathbb{R}_{\text {proj }}$ follows from Lemma 3.7 in [Spe99].

REMARK 4.3. Not every real closed field can be expanded to a model of $T$. Indeed, the only integer part of the field of real algebraic numbers $\mathbb{R}_{\text {alg }}$ is $\mathbb{Z}$. In $\left(\mathbb{R}_{\text {alg }}, \mathbb{Z}\right)$, however, we can define the cut below any transcendental number that has a recursive decimal expansion - e.g. the constant $e$. Consequently, $\left(\mathbb{R}_{\mathrm{alg}}, \mathbb{Z}\right)$ is not definably connected.

We conclude this section with a lemma that is used below.

Lemma 4.4. If $\left(W_{\alpha}\right)_{\alpha \in J}$ is a decreasing definable sequence of closed and bounded nonempty subsets of $R^{n}$, then $\bigcap_{\alpha} W_{\alpha}$ is nonempty. 
Proof. As this argument is similar to the proof of Lemma 1.9 in [Mil01], we provide only a sketch. Using a lexicographical ordering and definable completeness, we can show that there is definable function $g: \mathcal{N} \rightarrow R^{n}$ such that $g(\alpha) \in W_{\alpha}$ for all $\alpha$. By definable completeness again, the range of this function has a limit point, which lies in $\bigcap_{\alpha} W_{\alpha}$.

5. o-minimality of relative Pfaffian closure. The proof of Theorem 1.6 follows [Spe99] very closely, and although superficial adjustments are necessary throughout, it would be tedious and uninstructive to list them all. Instead, we zoom in on two spots in the proof that illustrate the general difficulties and their remedies. Let us recall the context.

Inspired by the methods of [LR98], the proof of o-minimality in [Spe99] is axiomatic; rather than working with $\widetilde{\mathcal{R}}$-Pfaffian sets directly, the properties that make the proof work are isolated through a system of $\Lambda$-sets. In parallel with that development, we fix a system $\Lambda=\left(\Lambda_{n}\right)_{n \in \mathbb{N}}$ of collections $\Lambda_{n}$ of definable subsets of $R^{n}$. A subset $W$ of $R^{n}$ is called a $\Lambda$-set if $W \in \Lambda_{n}$ for some $n$. We also assume the following seven axioms for each $n$ and each set $W$ in $\Lambda_{n}$ :

(I) If a subset $Z$ of $R^{n}$ is definable in $\overline{\mathcal{R}}$, then $Z$ is in $\Lambda_{n}$.

(II) If $Z \in \Lambda_{n}$, then $W \cap Z$ is in $\Lambda_{n}$.

(III) $W \times R$ is in $\Lambda_{n+1}$.

(IV) If $\pi$ is a permutation of $\{1, \ldots, n\}$, then $\pi(W):=\left\{\left(x_{\pi(a)}, \ldots, x_{\pi(n)}\right)\right.$ : $x \in W\}$ is in $\Lambda_{n}$.

(V) There is a natural number $m$ greater than $n$ and a closed $W^{\prime}$ in $\Lambda_{m}$ such that $W=\Pi_{n}\left(W^{\prime}\right)$.

(VI) If $1 \leq k \leq n$, then there is a natural number $K$ such that for each $z$ in $R^{k}$ the fiber $W_{z}$ has fewer than $K$ components.

(VII) There is a natural number $K$ and sets $W^{1}, \ldots, W^{K}$ in $\Lambda_{n}$ such that $W=\bigcup_{p=1}^{K} W^{p}$ and each $W^{p}$ is an $\mathcal{R}$-manifold in standard position.

Definition 5.1. Let $W$ be a definable subset of $R^{k} \times R^{m} \times R^{l}$, and let $\varepsilon: \mathcal{N}^{2} \rightarrow R^{k}$ be a definable function. A subset $X$ of $R^{m}$ is obtained from $W$ if the following conditions hold:

(i) For each pair $(\alpha, \beta)$, the fiber $W_{\varepsilon(\alpha, \beta)}$ is a closed and bounded subset of $R^{m} \times R^{l}$.

(ii) For each $\alpha$ in $\mathcal{N}$, the sequence $(W(\alpha, \beta))_{\beta}$ of subsets of $R^{m}$ is decreasing, where $W(\alpha, \beta):=\Pi_{m}\left(W_{\varepsilon(\alpha, \beta)}\right)$.

(iii) The sequence $(W(\alpha))_{\alpha}$ is increasing, where $W(\alpha):=\bigcap_{\beta} W(\alpha, \beta)$.

(iv) Finally, $X=\bigcup_{\alpha} W(\alpha)$.

When a set $X$ is obtained from a $\Lambda$-set $W$, we call $X$ a basic $\Lambda^{\infty}$-set. A $\Lambda^{\infty}$-set is a finite union of basic $\Lambda^{\infty}$-sets. 
Remarks: In the case where $\mathcal{R}=\mathbb{R}_{\text {proj }}$, this definition agrees with the definition in [Spe99]. Also, since the function $\varepsilon$ is required to be definable, each $\Lambda^{\infty}$-set is definable too.

Let $\mathcal{I}$ be the closed interval $[-1,1]$. The $\Lambda^{\infty}$-sets are used to build an o-minimal structure on $\mathcal{I}$. We list some lemmas to set the scene. The labels in parentheses refer to the analogous results in [Spe99].

Lemma 5.2 (Cor. 2.9 and Lemma 2.11). The collection of $\widetilde{\mathcal{R}}$-Pfaffian sets satisfies Axioms (I)-(VII) in the definition of $\Lambda$-sets.

Lemma 5.3 (Lemma 3.2). Every $\Lambda$-set is a $\Lambda^{\infty}$-set.

Lemma 5.4 (Proposition 3.3). Let $W$ be a $\Lambda$-set. Then there is a natural number $K$ such that every basic $\Lambda^{\infty}$-set $X$ obtained from $W$ has fewer than $K$ components.

Note: The bound in the previous lemma does not depend on the function $\varepsilon: \mathcal{N}^{2} \rightarrow R^{k}$ used to obtain $X$.

Lemma 5.5 (Proposition 3.4, Remark on p. 200, and Remark 3.5).

(a) The collection of $\Lambda^{\infty}$-sets is closed under taking finite unions, cartesian products, permutations of coordinates, and coordinate projections.

(b) The collection of $\Lambda^{\infty}$-sets is closed under taking fibers. In fact, if $X$ is obtained from $W$, then the fiber $X_{a}$ is also obtained from $W$.

(c) The collection of $\Lambda^{\infty}$-sets is closed under taking finite intersections. In fact, if $X^{\prime}$ is obtained from $W^{\prime}$, then $X \cap X^{\prime}$ is obtained from a $\Lambda$-set that depends on $W$ and $W^{\prime}$ but not on $X$ nor $X^{\prime}$.

Part (c) together with Lemma 5.4 shows:

Corollary 5.6. Let $W$ and $W^{\prime}$ be $\Lambda$-sets. Then there is a natural number $K$ such that whenever $X$ and $X^{\prime}$ are $\Lambda^{\infty}$-subsets of $R^{n}$ obtained from $W$ and $W^{\prime}$ respectively, then $X \cap X^{\prime}$ has fewer than $K$ components.

The difficult part of showing that the $\Lambda^{\infty}$-subsets of cartesian powers of $\mathcal{I}$ form an o-minimal structure on $\mathcal{I}$ is to show that if $X$ is a $\Lambda^{\infty}$-subset of $\mathcal{I}^{m}$, then $\mathcal{I} \backslash X$ is also a $\Lambda^{\infty}$-set. This is done by induction on $m$ (see Proposition 3.12 of [Spe99] for the precise statement). The idea is to show that the boundary of $X$ is contained in a set $Y$ that can be partitioned into graphs of continuous functions with $\Lambda^{\infty}$-domains. One can then recover both $X$ and $\mathcal{I}^{m} \backslash X$ as disjoint unions of $\Lambda^{\infty}$-sets. Morally speaking, the process is like producing a cell-decomposition of $X$ from scratch and then assembling $\mathcal{I}^{m} \backslash X$ from the resulting cells.

The set $Y$ mentioned in the last paragraph is described in terms of Hausdorff limits, and to show that $Y$ has empty interior is a preliminary and delicate task. The first lemma that we prove in depth (corresponding 
to Lemma 3.8 from [Spe99]) is used for this purpose. The complication that arises in our setting is that an $\mathcal{R}$-finite set may be infinite. Thus we have to take care that our counting is done in a definable way.

Before we begin, let us mention two predictable facts: (1) Every $\mathcal{R}$-finite set $X$ is bounded, and (2) if a set $X$ is $\mathcal{R}$-infinite, then for each $\beta$ in $\mathcal{N}$ there is a definable injection $f:\{0, \ldots, \beta\} \rightarrow X$. These can be proved by induction.

Lemma 5.7. Let $m>1$, let $W$ be a $\Lambda$-set, and let $(Y(\alpha))_{\alpha}$ be a definable sequence of $\Lambda^{\infty}$-sets in $\mathcal{I}^{m}$ obtained from $W$. Suppose that $(Y(\alpha))_{\alpha}$ converges to a Hausdorff limit $Y$. Then the set

$$
B:=\left\{a \in R: Y_{a} \neq \emptyset \text { and } Y_{a} \text { is not a Hausdorff limit of }\left(Y(\alpha)_{a}\right)_{\alpha}\right\}
$$

is $\mathcal{R}$-countable.

Proof. By axiom (H), we can write $B=\bigcup_{U} B_{U}$ where $U$ ranges over all terminating definable sequences $\left(U_{\gamma}\right)_{\gamma<\beta}$ of $\mathcal{R}$-rational boxes in $\mathcal{I}^{m-1}$ and

$$
B_{U}=\{a \in R \text { : condition }(*) \text { from axiom }(\mathrm{H}) \text { holds for } a\} .
$$

Note that the collection of all such $\left(U_{\gamma}\right)_{\gamma}$ is definable and $\mathcal{R}$-countable. Fix $\left(U_{\gamma}\right)_{\gamma<\beta}$. From Corollary 5.6, it follows that there is a natural number $K$ such that $Y(\alpha) \cap\left(\mathcal{I} \times U_{\gamma}\right)$ has at most $K$ components for all $\alpha, U$, and $\gamma$. We will show that there is no definable injection $f:\{0, \ldots, 2 K \beta\} \rightarrow B_{U}$, which by fact (2) preceding the lemma shows that $B_{U}$ is $\mathcal{R}$-finite. It follows that $B_{U}$-and hence $B$-is $\mathcal{R}$-countable.

Assume for a contradiction that there is a definable sequence $\left(a_{\delta}\right)_{\delta \leq 2 K \beta}$ such that each $a_{\delta} \in B_{U}$ and $a_{\delta_{1}} \neq a_{\delta_{2}}$ whenever $\delta_{1} \neq \delta_{2}$. By fact (1) preceding the lemma, the definable set $\left\{\left|a_{\delta_{1}}-a_{\delta_{2}}\right|: \delta_{1}<\delta_{2} \leq 2 K \beta\right\}$ is bounded away from 0 . Consequently, there is an $s \in R$ such that $s>0$ and the intervals $I_{\delta}:=\left(a_{\delta}-s, a_{\delta}+s\right)$ are pairwise disjoint.

The first part of condition (*) for $a_{\delta}$ says that $Y_{a_{\delta}} \cap U_{\gamma} \neq \emptyset$ for each $\gamma<\beta$. Because $Y$ is the limit of the sets $Y(\alpha)$, we have $Y(\alpha) \cap\left(I_{\delta} \times U_{\gamma}\right) \neq \emptyset$ for all sufficiently large $\alpha$. By the second part of condition $(*)$ for $a_{\delta}$, there is a $\gamma(\delta)$ such that $Y(\alpha)_{a_{\delta}} \cap U_{\gamma(\delta)}=\emptyset$ for sufficiently large $\alpha$.

Since there are only $\mathcal{R}$-finitely many such $\delta$, by fact (1) above we can find a single $\alpha^{\prime}$ such that for each $\delta \leq 2 K \beta$ :

(1) $Y\left(\alpha^{\prime}\right) \cap\left(I_{\delta} \times U_{\gamma}\right) \neq \emptyset$ for each $\gamma<\beta$,

(2) there is a $\gamma(\delta)$ such that $Y\left(\alpha^{\prime}\right)_{a_{\delta}} \cap U_{\gamma(\delta)}=\emptyset$.

Now axiom (B) implies that there is a $\gamma^{\prime}<\beta$ and a definable injection $g:\{0, \ldots, 2 K\} \rightarrow\{0, \ldots, 2 K \beta\}$ such that $Y\left(\alpha^{\prime}\right)_{a_{g(\kappa)}} \cap U_{\gamma^{\prime}}=\emptyset$ for all $\kappa$ in $\{0, \ldots, 2 K\}$. 
It follows that for each component $C$ of $Y\left(\alpha^{\prime}\right) \cap\left(\mathcal{I} \times U_{\gamma^{\prime}}\right)$ there are at most two numbers $\kappa$ such that $Y\left(\alpha^{\prime}\right) \cap\left(I_{g(\kappa)} \times U_{\gamma^{\prime}}\right)$ meets $C$. This means that $Y\left(\alpha^{\prime}\right) \cap\left(\mathcal{I} \times U_{\gamma^{\prime}}\right)$ has at least $K+1$ components, a contradiction.

REMARK 5.8. Although the sequence $(Y(\alpha))_{\alpha}$ is required to be definable and each $Y(\alpha)$ is obtained from $W$, we did not need to know whether the functions $\varepsilon_{\alpha}: \mathcal{N}^{2} \rightarrow R^{k(\alpha)}$ used to obtain $Y(\alpha)$ are uniformly definable.

Fix a $\Lambda^{\infty}$-subset $X$ of $\mathcal{I}^{m}$. The other lemma that we focus on is as follows:

Lemma 5.9. There is a closed $\Lambda^{\infty}$-subset $Y$ of $\mathcal{I}^{m}$ such that $\operatorname{int}(Y)=\emptyset$ and $\operatorname{bd}(X) \subseteq Y$.

In fact, we only prove one claim in the lemma, whose proof requires several choices of infinite sequences. Again, we must take care to do this in a definable way.

Proof. We may assume that $X$ is a nonempty basic $\Lambda^{\infty}$-set obtained from a subset $W$ of $R^{k} \times \mathcal{I}^{m} \times R^{l}$. The set $Y$ we are looking for is obtained with Hausdorff limits as follows:

Using axiom (VII) for $\Lambda$-sets, we find $W^{1}, \ldots, W^{K}$ in $\Lambda_{n}$ such that $W=$ $\bigcup_{p=1}^{K} W^{p}$ and each $W^{p}$ is an $\mathcal{R}$-manifold in standard position. In particular, each nonempty fiber $W_{\varepsilon}^{p}$ with $\varepsilon$ in $R^{k}$ is an $\mathcal{R}$-manifold in standard position. Moreover, there is a natural number $d_{p}$ independent of $\varepsilon$ such that whenever $W_{\varepsilon}^{p}$ is nonempty the map $\left.\Pi_{m}\right|_{W_{\varepsilon}^{p}}$ has constant rank $d_{p}$.

For each $\alpha, \beta$, and $p=1, \ldots, K$, we put $Y^{p}(\alpha, \beta):=\Pi_{m}\left(\operatorname{cl}\left(W_{\varepsilon(\alpha, \beta)}^{p}\right)\right)$ in the notations of Definition 5.1. Then we apply $(\mathrm{G})$ to get unbounded definable subsets $I$ and $J$ of $\mathcal{N}$ such that the following hold:

(i) For each $p$ and $\alpha \in I$, the sequence $\left(Y^{p}(\alpha, \beta)\right)_{\beta \in J}$ converges to the Hausdorff limit $Y^{p}(\alpha)$ if this sequence is nonterminating. Otherwise we put $Y^{p}(\alpha)=\emptyset$.

(ii) For each $p$, the sequence $\left(Y^{p}(\alpha)\right)_{\alpha \in I}$ converges to a Hausdorff limit $Y^{p}$ if this sequence is nonterminating. Otherwise put $Y^{p}=\emptyset$.

Finally, we set $Y:=\bigcup_{p \in S} Y^{p}$ where

$$
S:=\left\{p \in\{1, \ldots, K\}: d_{p}<m\right\} .
$$

The claim we scrutinize is as follows:

Claim 1. For each $p$ in $S$, there are a natural number $n(p) \geq n$ and $a$ $\left.\Lambda_{n(p)}\right)^{\text {-set }} \widetilde{W}^{p}$ such that the sets $Y^{p}(\alpha)$ and $Y^{p}$ are basic $\Lambda^{\infty}$-sets obtained from $\widetilde{W}^{p}$. In particular, $Y$ is a $\Lambda^{\infty}$-set.

To finish the lemma one also needs

Claim 2. $Y$ contains bd $(X)$. 
Claim 3. Y has empty interior.

Since $Y$ is closed, Claim 2 can be proved by showing that for each $a$ in $\operatorname{bd}(X)$ and $t>0$ we have $d(a, Y)<t$. In this way we can avoid talking about $a$ as the limit of a converging sequence as done in [Spe99]. Claim 3 involves Lemma 5.7 above and significant use of the rank theorem, but the arguments used are the same. Here is the proof of Claim 1:

For each $\alpha$ in $I$, each $\beta$ in $J$, and each $p$ in $S$ it is convenient to define

$$
\begin{array}{r}
\varrho_{p}(\alpha, \beta):= \begin{cases}d\left(Y^{p}(\alpha, \beta), Y^{p}(\alpha)\right) & \text { if } Y^{p}(\alpha) \neq \emptyset \text { and } Y^{p}(\alpha, \beta) \neq \emptyset, \\
0 & \text { if } Y^{p}(\alpha)=\emptyset \text { and } Y^{p}(\alpha, \beta)=\emptyset, \\
1 & \text { otherwise; }\end{cases} \\
\varrho_{p}(\alpha):= \begin{cases}d\left(Y^{p}(\alpha), Y^{p}\right) & \text { if } Y^{p}(\alpha) \neq \emptyset \text { and } Y^{p} \neq \emptyset, \\
0 & \text { if } Y^{p}(\alpha)=\emptyset \text { and } Y^{p}=\emptyset, \\
1 & \text { otherwise. }\end{cases}
\end{array}
$$

Notice that for each $p=1, \ldots, K$, we have $\lim _{\alpha \in I} \varrho_{p}(\alpha)=0$. Moreover, for each $p=1, \ldots, K$ and $\alpha$ in $I$, we have $\lim _{\beta \in J} \varrho_{p}(\alpha, \beta)=0$.

Now fix $p$ in $S$. To slightly simplify notation, we write $\varrho(\alpha)$ for $\varrho_{p}(\alpha)$ and $\varrho(\alpha, \beta)$ for $\varrho_{p}(\alpha, \beta)$. By Axiom $(\mathrm{V})$, there are a number $l^{\prime} \geq l$ and a closed set $V$ in $\Lambda_{k+m+l^{\prime}}$ such that $W^{p}=\Pi_{k+m+l}(V)$. Note that since $W_{\varepsilon(\alpha, \beta)}^{p}$ is bounded, for each $\alpha$ and $\beta$ we have

$$
Y^{p}(\alpha, \beta)=\Pi_{m}\left(\operatorname{cl}\left(W_{\varepsilon(\alpha, \beta)}^{p}\right)\right)=\operatorname{cl}\left(\Pi_{m}\left(W_{\varepsilon(\alpha, \beta)}^{p}\right)\right)=\operatorname{cl}\left(\Pi_{m}\left(V_{\varepsilon(\alpha, \beta)}\right)\right) .
$$

(The second equality follows from Proposition 2.1(v).)

Let $n(p):=k+1+1+m+m+l^{\prime}$, and let $x^{\prime}=\left(\theta, t, s, y, y^{\prime}, z^{\prime}\right)$ range over $R^{n(p)}$. Consider the $\Lambda$-set

$$
\widetilde{W}^{p}:=\left\{x^{\prime}:\left(\theta, y^{\prime}, z^{\prime}\right) \in V, d\left(y, y^{\prime}\right) \leq t, \text { and }\left\|z^{\prime}\right\| \leq s\right\} .
$$

Also define the set

$$
U:=\left\{x^{\prime}:\left(\theta, y^{\prime}, z^{\prime}\right) \in V, d\left(y, y^{\prime}\right)<t, \text { and }\left\|z^{\prime}\right\|<s\right\},
$$

and observe that for each $\alpha, \beta, \delta$, and $\varrho$ the set $\Pi_{m}\left(U_{\varepsilon(\alpha, \beta), \varrho, \delta}\right)$ is open in $R^{m}$. For each $\alpha, \beta$, and $\varrho$ such that $Y^{p}(\alpha, \beta)$ is nonempty, we have

$$
S\left(Y^{p}(\alpha, \beta), \varrho\right) \subset \bigcup_{\delta \in \mathcal{N}} \Pi_{m}\left(U_{\varepsilon(\alpha, \beta), 2 \varrho, \delta}\right) .
$$

To see this, choose $y \in S\left(Y^{p}(\alpha, \beta), \varrho\right)$. By definition, there is $y^{\prime \prime} \in Y^{p}(\alpha, \beta)$ such that $d\left(y, y^{\prime \prime}\right) \leq \varrho$. Thus by (5.1) above, there is $\left(y^{\prime}, z^{\prime}\right) \in V_{\varepsilon(\alpha, \beta)}$ such that $d\left(y, y^{\prime}\right)<2 \varrho$.

From Lemma 4.4, we deduce that for each $\alpha, \beta$, and $\varrho$ there is a $\gamma$ such that

$$
S\left(Y^{p}(\alpha, \beta), \varrho\right) \subset \Pi_{m}\left(U_{\varepsilon(\alpha, \beta), 2 \varrho, \gamma}\right) .
$$


Unraveling definitions, we also see that for all $\alpha, \beta, \delta$, and $\gamma$,

$$
\Pi_{m}\left(U_{\varepsilon(\alpha, \beta), \varrho, \delta}\right) \subseteq \Pi_{m}\left(\widetilde{W}_{\varepsilon(\alpha, \beta), \varrho, \delta}^{p}\right) \subseteq S\left(\Pi_{m} V_{\varepsilon(\alpha, \beta)}, \varrho\right) \subseteq S\left(Y^{p}(\alpha, \beta), \varrho\right) .
$$

We are now set up to prove the claim. First, fix $\alpha$ such that $Y^{p}(\alpha)$ is nonempty. Using (D), we may assume that $5 \varrho(\alpha, \beta+1)<\varrho(\alpha, \beta)$ for all $\beta$ in $\mathcal{N}$. For each $\beta$, define $\gamma(\beta)$ to be the least $\gamma$ such that

$$
S\left(Y^{p}(\alpha, \beta), 2 \varrho(\alpha, \beta)\right) \subset \Pi_{m}\left(U_{\varepsilon(\alpha, \beta), 4 \varrho(\alpha, \beta), \gamma(\beta)}\right)
$$

as given by (5.3). Then for all $\beta$ in $\mathcal{N}$,

$$
\begin{aligned}
S\left(Y^{p}(\alpha), \varrho(\alpha, \beta)\right) & \subseteq S\left(Y^{p}(\alpha, \beta), 2 \varrho(\alpha, \beta)\right) \subseteq \Pi_{m}\left(\widetilde{W}_{\varepsilon(\alpha, \beta), 4 \varrho(\alpha, \beta), \gamma(\beta)}^{p}\right) \\
& \subseteq S\left(Y^{p}(\alpha, \beta), 4 \varrho(\alpha, \beta)\right) \subseteq S\left(Y^{p}(\alpha), 5 \varrho(\alpha, \beta)\right) .
\end{aligned}
$$

It follows that $Y^{p}(\alpha)$ is the decreasing intersection of the definable sequence

$$
\left(\Pi_{m}\left(\widetilde{W}_{\varepsilon(\alpha, \beta), 4 \varrho(\alpha, \beta), \gamma(\beta)}^{p}\right)\right)_{\beta}
$$

of closed and bounded subsets of $R^{m}$.

Now assume that $Y^{p}$ is nonempty. Then by (D) we may assume that $8 \varrho(\alpha+1)<\varrho(\alpha)$ for all $\alpha$. Now for each $\alpha$, define $\beta(\alpha)$ to be the least $\beta$ satisfying $\varrho(\alpha, \beta)<\varrho(\alpha)$. Likewise, define $\gamma(\alpha)$ to be the least $\gamma$ satisfying

$$
S\left(Y^{p}(\alpha, \beta(\alpha)), 3 \varrho(\alpha)\right) \subset \Pi_{m}\left(U_{\varepsilon(\alpha, \beta(\alpha)), 6 \varrho(\alpha), \gamma(\alpha)}\right)
$$

as given by (5.3). Then for all $\alpha$ in $\mathcal{N}$,

$$
\begin{aligned}
S\left(Y^{p}, \varrho(\alpha)\right) & \subseteq S\left(Y^{p}(\alpha), 2 \varrho(\alpha)\right) \subseteq S\left(Y^{p}(\alpha, \beta(\alpha)), 3 \varrho(\alpha)\right) \\
& \subset \Pi_{m}\left(\widetilde{W}_{\varepsilon(\alpha, \beta(\alpha)), 6 \varrho(\alpha), \gamma(\alpha)}^{p}\right) \subseteq S\left(Y^{p}(\alpha, \beta(\alpha)), 6 \varrho(\alpha)\right) \\
& \subseteq S\left(Y^{p}(\alpha), 7 \varrho(\alpha)\right) \subseteq S\left(Y^{p}, 8 \varrho(\alpha)\right) .
\end{aligned}
$$

This means that $Y^{p}$ is the decreasing intersection of the closed and bounded sets $\left(\Pi_{m}\left(\widetilde{W}_{\varepsilon(\alpha, \beta(\alpha)), 6 \varrho(\alpha), \gamma(\alpha)}^{p}\right)\right)_{\alpha}$. This finishes the proof of the claim.

6. Uniform bounds on components for $\mathfrak{P}(\widetilde{\mathbb{R}})$. Finally, we prove Theorem 1.8. The proof is basically a model-theoretic compactness argument using Theorem 1.6 and a nonstandard model of $T_{\text {proj. There remain }}$ a few details that need to be checked, and we begin with some elementary observations. The first two propositions in this section hold for an arbitrary extension $T$ of the theory of dense linear orders. Let $\mathcal{R}$ be a model of $T$.

Proposition 6.1. Let $\phi(x, y)$ be a parameter-free formula, and let $K$ be a natural number such that for all $b$ in $R^{m}$ the set $\phi\left(R^{n}, b\right)$ has fewer than $K$ components. Then for any structure $\mathcal{S}$ that is elementarily equivalent to $\mathcal{R}$, and any $b^{\prime}$ in $S$, the set $\phi\left(S^{n}, b^{\prime}\right)$ has fewer than $K$ components. 
Proof. Let $\mathcal{S}$ be elementarily equivalent to $\mathcal{R}$, and suppose for a contradiction that the set $X_{b^{\prime}}:=\phi\left(S^{n}, b^{\prime}\right)$ has at least $K$ components for some $b^{\prime}$ in $S^{m}$. Then there are $K$ parameter-free definable families $U_{z}^{1}, \ldots, U_{z}^{K}$ of subsets of $S^{n}$ and a $c$ in $S^{l}$ such that

(1) each $U_{c}^{i}$ is an open subset of $S^{n}$,

(2) the sets $U_{c}^{i}$ are pairwise disjoint,

(3) $X_{b^{\prime}} \subseteq \bigcup_{i=1}^{K} U_{c}^{i}$

(4) for each $i=1, \ldots, K$ the set $U_{c}^{i} \cap X_{b^{\prime}}$ is not empty.

One can write down a first-order parameter-free formula that expresses that there are $b^{\prime}$ and $c$ satisfying all of these conditions. This formula must hold in $\mathcal{R}$, a contradiction.

Definition 6.2. Suppose $\mathcal{P}$ is a property of sets. We say $\mathcal{P}$ is $T$-absolute if for each parameter-free formula $\phi(x, y)$ there is another parameter-free formula $d_{\phi}(y)$ such that for each model $\mathcal{S}$ of $T$ and all $c$ in $S^{m}$ we have the following:

The set $\phi\left(S^{n}, c\right)$ has property $\mathcal{P}$ if and only if $\mathcal{S} \models d_{\phi}(c)$.

For $T$-absoluteness of definable connectedness, we have an equivalence:

Proposition 6.3. The following are equivalent:

(1) Definable connectedness is T-absolute.

(2) For each formula $\phi(x, y)$ there is a formula $\psi_{\phi}(x, y)$ such that for all $c$ in $R^{m}$, the set $\phi\left(R^{n}, c\right)$ is not definably connected if and only if $\psi_{\phi}\left(R^{n}, c\right)$ is a proper nonempty closed and open subset of $\phi\left(R^{n}, c\right)$.

Proof. For $(1) \Rightarrow(2)$ suppose that definable connectedness is $T$-absolute. Let $\phi(x, y)$ be a formula, and suppose for a contradiction that there is no corresponding $\psi_{\phi}(x, y)$. Let the formula $d_{\phi}(y)$ be as in the definition of $T$-absoluteness, and let $\chi_{\psi}(y)$ be a formula expressing that $\psi\left(R^{n}, y\right)$ is a nonempty proper closed and open subset of $\phi\left(R^{n}, y\right)$. Then the type

$$
\Phi:=\left\{\neg \chi_{\psi}(y): \psi \text { an } \mathfrak{L}_{\text {proj-formula }} \cup\left\{\neg d_{\phi}(y)\right\}\right.
$$

is consistent. Consequently, this type is realized in some model of $T$ by the compactness theorem, a contradiction.

For the converse, simply take $d_{\phi}(y)$ to be $\neg \chi_{\psi}(y)$.

From now on we assume that $\mathcal{R}$ is a model of $T_{\text {proj }}$. We note first that definable connectedness is $T_{\text {proj-absolute. }}$

Lemma 6.4. Let $K$ be a natural number. The following property $\mathcal{P}$ is $T_{\text {proj-absolute: }}$

- $\mathcal{P}(X):=$ "X has at least $K$ components." 
Proof. Let $Y^{n}$ be the definable subset of $R^{n+1}$ given by Proposition 4.1, the fibers of which are precisely the open subsets of $R^{n}$. For a formula $\phi(x, y)$, let $d_{\phi}(y)$ be a formula expressing that there is a $K$-tuple $\left(r_{1}, \ldots, r_{K}\right)$ in $R^{K}$ such that the sets $Y_{r_{i}}^{n}$ are pairwise disjoint, each intersecting $\phi\left(R^{n}, y\right)$, and their union covers $\phi\left(R^{n}, y\right)$.

Corollary 6.5. Suppose $\mathcal{R}$ is $\omega$-saturated. Suppose also that $X_{y}$ is a definable family such that each fiber $X_{b}$ has only finitely many components. Then there is a natural number $K$ such that each fiber has fewer than $K$ components.

Proof. By the preceding lemma, there is a formula $d^{k}(y)$ such that $\mathcal{R} \models d^{k}(c)$ if and only if $X_{c}$ has at least $K$ components. Then consider the type $\left\{d^{k}(y): k \in \mathbb{N}\right\}$.

Now we check $T_{\text {proj-absoluteness up to the Rolle property. }}$

Lemma 6.6. Fix natural numbers $m$ and $k$. Then the following property $\mathcal{P}$ is $T_{\text {proj-absolute: }}$

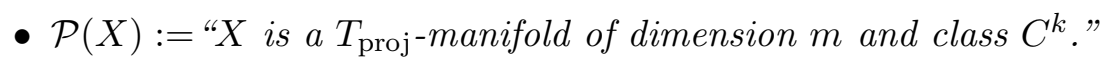

Proof. Using Proposition 4.1, there is a first-order formula $d_{\phi}(y)$ expressing the following:

"For every $x$ in $\phi\left(R^{n}, y\right)$, there is a chart $\varphi$ for $\phi\left(R^{n}, y\right)$ at $x . "$

Then $d_{\phi}(y)$ satisfies our requirements.

Similarly we obtain:

Lemma 6.7. Let $\left(\omega_{z}\right)_{z \in R}$ be a parameter-free definable family of 1-forms on a definable family $\left(U_{z}\right)_{z \in R}$ of open sets. Then the following properties are $T_{\text {proj-absolute: }}$

- $\mathcal{P}(X):=$ "There is a parameter $c$ such that $X$ is an integral $T_{\text {proj- }}$ manifold of $\omega_{c}=0$."

- $\mathcal{P}(X):=$ "There is a parameter $c$ such that $X$ is a Rolle $T_{\text {proj-leaf of }}$ $\omega_{c}=0 . "$

Proof. By Proposition 4.1, Lemma 6.4, and Lemma 6.6 we can definably quantify over all definably connected $\mathcal{R}$-manifolds of dimension 1 and class $C^{1}$.

We conclude by deducing Theorem 1.8 from Theorem 1.6. In fact, we prove a slightly more general version after modestly extending the definitions.

Let $\widetilde{\mathfrak{L}}$ be the language of some o-minimal structure $\widetilde{\mathcal{S}}$ that expands a real closed field and has an expansion to a model $\mathcal{S}$ of $T_{\text {proj. }}$. Let $P_{1}, \ldots, P_{j}$ 
be predicate symbols none of which is in $\mathfrak{L}_{\text {proj }}$ or in $\widetilde{\mathfrak{L}}$. Let $\Phi=\left(\phi_{0}, \ldots, \phi_{j}\right)$ be a tuple of $\widetilde{\mathfrak{L}}$-formulas.

Definition 6.8. We say that a subset $X$ of $S^{n}$ has format $\Phi$ if there is an interpretation of $P_{1}, \ldots, P_{j}$ such that the following hold:

(1) Each $\phi_{i}$ is in the language $\widetilde{\mathfrak{L}} \cup\left\{P_{1}, \ldots, P_{i}\right\}$.

(2) For $i=0, \ldots, j-1$, each $\phi_{i}$ defines (in $\mathcal{S}$ ) the graph of a nonvanishing vector field on an open set $U_{i}$.

(3) For $i=0, \ldots, j-1$, each $P_{i+1}$ defines an $\mathcal{S}$-Rolle leaf of the nonsingular 1-form determined by $\phi_{i}$.

(4) The set $X$ is defined by the formula $\phi_{j}$.

Here is the generalization of Theorem 1.8 that we prove:

THEOREM 6.9. There is a natural number $K$ such that whenever

(i) $\widetilde{\mathcal{S}}^{\prime}$ is a structure that is elementarily equivalent to $\widetilde{\mathcal{S}}$,

(ii) $\widetilde{\mathcal{S}}^{\prime}$ has an expansion $\mathcal{S}^{\prime}$ to a model of $T_{\text {proj }}$,

(iii) $X$ is a set that is definable in $\mathfrak{P}\left(\widetilde{\mathcal{S}}^{\prime}, \mathcal{S}^{\prime}\right)$,

(iv) $X$ has format $\Phi$,

then the set $X$ has fewer than $K$ components (with respect to $\mathcal{S}^{\prime}$ ).

In other words, the bound $K$ does not even depend on the structure $\widetilde{\mathcal{S}}$.

Proof. We first introduce a notational convention. For each $i=1, \ldots, j$,

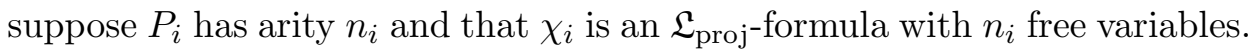
We write $\phi_{i}\left[\chi_{1}, \ldots, \chi_{i-1}\right]$ for the formula obtained from $\phi_{i}$ by replacing, for each $k=1, \ldots, i-1$, each occurrence of $P_{k}(x)$ by $\chi_{k}(x)$, making sure that free variables are consistently replaced in each occurrence. We also use $x^{1}, x^{2}, \ldots$ to denote tuples of variables.

Fix a format $\Phi$. Let $\mathcal{R}$ be an $\omega$-saturated elementary extension of $\mathcal{S}$, and assume first that for each $K$ there is an $\mathcal{R}$-definable set $Y^{K}$ with format $\Phi$ and at least $K$ components. By Proposition 4.1 and Lemma 6.7, for each $i=1, \ldots, j-1$ there is a parameter-free formula $\chi_{i}\left(x^{i}, t\right)$ such that the set $\left\{\chi_{i}\left(R^{n_{i}}, r\right): r \in R\right\}$ is equal to the collection of all $\mathcal{R}$-Rolle leaves of all 1 -forms corresponding to the definable vector fields

$$
\phi_{i}\left[\chi_{1}\left(x^{1}, c^{1}\right), \ldots, \chi_{i-1}\left(x^{i-1}, c^{i-1}\right)\right]\left(x^{i}\right)
$$

as the parameters $c^{1}, \ldots, c^{i-1}$ vary. By Corollary 6.5 , there are in fact parameters $c^{1}, \ldots, c^{j-1}$ such that the formula

$$
\phi_{j}\left[\chi_{1}\left(x^{1}, c^{1}\right), \ldots, \chi_{j-1}\left(x^{j-1}, c^{j-1}\right)\right]\left(x^{j}\right)
$$

defines a set $Y$ with infinitely many components and also such that $\chi_{i}\left(x^{i}, c^{i}\right)$ defines a Rolle $\mathcal{R}$-leaf of a 1 -form determined by (6.1) for $i=1, \ldots, j-1$. 
Hence $Y$ is definable in the o-minimal structure $\mathfrak{P}\left(\left.\mathcal{R}\right|_{\mathfrak{L}}, \mathcal{R}\right)$. This contradiction shows that there is a $K$ such that every $\mathcal{R}$-definable set with format $\Phi$ has fewer than $K$ components.

For the general case, notice that every $\mathcal{S}^{\prime}$-definable set with format $\Phi$ is defined by formula (6.2) for some choice of parameters $c^{1}, \ldots, c^{j-1}$. The result now follows from Proposition 6.1.

Acknowledgments. The work in this paper is derived from the author's doctoral dissertation prepared at McMaster University under the supervision of Patrick Speissegger. Additional thanks go to Bradd Hart, Deirdre Haskell, David Marker, and Matt Valeriote, for helpful comments on this work while still in the form of a thesis. Special thanks also to Alf Dolich and Chris Miller for insightful suggestions at critical stages. Finally, thanks to the referee whose attentive reading uncovered a significant error in an earlier version. Any remaining errors are the author's alone.

\section{References}

[vdD98] L. van den Dries, Tame Topology and o-Minimal Structures, London Math. Soc. Lecture Note Ser. 248, Cambridge Univ. Press, Cambridge, 1998.

[Gab03] A. Gabrielov, Relative closure and the complexity of Pfaffian elimination, in: Discrete and Computational Geometry, Algorithms Combin. 25, Springer, Berlin, 2003, 441-460.

[Kec95] A. S. Kechris, Classical Descriptive Set Theory, Grad. Texts in Math. 156, Springer, New York, 1995.

[Kho91] A. G. Khovanskiı̌, Fewnomials, Transl. Math. Monogr. 88, Amer. Math. Soc., Providence, RI, 1991.

[KM99] M. Karpinski and A. Macintyre, A generalization of Wilkie's theorem of the complement, and an application to Pfaffian closure, Selecta Math. (N.S.) 5 (1999), 507-516.

[KS05] S. Kuhlmann and S. Shelah, $\kappa$-bounded exponential-logarithmic power series fields, Ann. Pure Appl. Logic 136 (2005), 284-296.

[LR98] J.-M. Lion et J.-P. Rolin, Volumes, feuilles de Rolle de feuilletages analytiques et théorème de Wilkie, Ann. Fac. Sci. Toulouse Math. (6) 7 (1998), 93-112.

[Mil01] C. Miller, Expansions of dense linear orders with the intermediate value property, J. Symbolic Logic 66 (2001), 1783-1790.

[MR93] M.-H. Mourgues and J. P. Ressayre, Every real closed field has an integer part, J. Symbolic Logic 58 (1993), 641-647.

[MR91] R. Moussu et C. Roche, Théorie de Hovanskiı et problème de Dulac, Invent. Math. 105 (1991), 431-441.

[Mun75] J. R. Munkres, Topology: a First Course, Prentice-Hall, Englewood Cliffs, NJ, 1975.

[OPP96] M. Otero, Y. Peterzil, and A. Pillay, On groups and rings definable in o-minimal expansions of real-closed fields, Bull. London Math. Soc. 28 (1996), 7-14.

[PS86] A. Pillay and Ch. Steinhorn, Definable sets in ordered structures, I, Trans. Amer. Math. Soc. 295 (1986), 565-592.

[Roy88] H. L. Royden, Real Analysis, 3rd ed., Macmillan, New York, 1988. 
[Spe99] P. Speissegger, The Pfaffian closure of an o-minimal structure, J. Reine Angew. Math. 508 (1999), 189-211.

[Wil99] A. J. Wilkie, A theorem of the complement and some new o-minimal structures, Selecta Math. (N.S.) 5 (1999), 397-421.

Division of Natural Sciences and Mathematics

The College of New Rochelle

29 Castle Place

New Rochelle, NY 10805, U.S.A.

E-mail: sfratarcangeli@cnr.edu

Received 3 November 2006;

in revised form 24 December 2007 